\title{
Improved two-point model for limiter scrape-off layer
}

\author{
M. Z. Tokar \\ Institut für Plasmaphysik, Forschungszentrum Jülich GmbH, Association FZJ-Euratom, \\ 52425 Jülich, Germany \\ M. Kobayashi \\ National Institute for Fusion Science, Toki-shi, Gifu-ken 509-5292, Japan \\ Y. Feng \\ Max-Planck-Institut für Plasmaphysik, Euratom Association, D-17491 Greifswald, Germany
}

(Received 29 March 2004; accepted 15 July 2004; published online 8 September 2004)

\begin{abstract}
An analytical model for a limiter scrape-off layer (SOL) is proposed, which takes self-consistently into account both conductive and convective contributions to the heat transport in SOL. The particle flows in the SOL main part are determined by considering the recycling of neutrals. The model allows us to interpret the results of numerical simulation by the code EMC3-EIRENE [Y. Feng, F. Sardei, P. Grigull, K. McCormick, J. Kisslinger, D. Reiter, and Y. Igitkhanov, Plasma Phys. Controlled Fusion 44, 611 (2002)] for the edge region of Tokamak Experiment for Technology Oriented Research (TEXTOR) [Proceedings of the 16th IEEE Symposium on Fusion Engineering, 1995 (Institute for Electrical and Electronics Engineers, Piscataway, NJ, 1995), p. 470]. (C) 2004 American Institute of Physics. [DOI: 10.1063/1.1791232]
\end{abstract}

\section{INTRODUCTION}

In fusion devices the confined plasma volume with nested closed magnetic surfaces is screened from the wall by the so-called scrape-off layer (SOL) where magnetic field lines can hit the wall elements. ${ }^{1,2}$ In a divertor configuration the magnetic topology is principally different in these two regions, which are separated by a separatrix created by currents in special coils. In a tokamak with a mechanical limiter the last closed magnetic surface (LCMS) is the outmost one, which first touches the limiter.

The plasma parameters in SOL are very important for characterization of both the plasma wall interaction and the plasma behavior in confined volume. Normally complex two- or three-dimensional transport codes are used to compute parameter profiles along and perpendicular to the magnetic field (see, e.g., Refs. 3-6). However, reduced analytical models are also often applied for rough estimates and in order to gain a qualitative insight into physics of transport processes in SOL. The so-called 2-point model, ${ }^{1,2}$ which is based on the assumption that the heat transport parallel to the magnetic field is of purely conductive nature, is normally relevant to a divertor SOL. This approach is validated by the fact that the particle flows generated by the ionization of recycling neutrals are localized very close to the divertor plates and do not contribute significantly to the energy transfer in the main part of the SOL. Conversely in limiter devices, the neutralization of charged particles occurs very close to the LCMS and produced neutrals can easily penetrate into the confined volume. The ions and electrons produced after ionization of neutrals spread over magnetic surfaces and diffuse perpendicular to the magnetic field back into the SOL where they stream to the limiter. This particle flow loop schematically shown in Fig. 1(a) for the case of a plasma bounded by a toroidally symmetric bumper limiter can be very important for the SOL power balance. In the present paper we amend the 2-point model for SOL by including self-consistently convective heat transport into consideration. It is demonstrated that the improved model adequately describes a limiter SOL in agreement with the results of numerical modeling with 3D code EMC3-EIRENE. ${ }^{6,7}$ A comparison with the standard 2-point model corrected with respect to convective energy transport ${ }^{1}$ is provided.

\section{BASIC MODEL EQUATIONS}

\section{A. Main part of SOL}

In the case of a bumper limiter in Fig. 1(a) the angle $\varepsilon$, at which the field lines contact the surface, varies with the distance from the poloidal plane. In the present analytical consideration we replace this limiter shape by a "roof-top," one shown in Fig. 1(b), with $\varepsilon=$ const. The fact that the results do not depend explicitly on $\varepsilon$ (for $\varepsilon \ll 1$ assumed) allows us to apply them, to a certain extend, to the more realistic limiter geometry in Fig. 1(a). The limiter SOL can be divided into two zones [see Fig. 1(b)] where different transport processes are of importance. In the main part of SOL the particle source due to neutral ionization is small and particle transport is described by the following continuity equation:

$$
\frac{d \Gamma_{\|}}{d l}=\frac{\Gamma_{\perp}^{L C M S}}{\delta} .
$$

Here $\Gamma_{\|}$is the component of particle flux density along the magnetic field (direction $l$ ), $\Gamma_{\perp}^{L C M S}$ is the density of charged particle influx into SOL from the confined plasma volume perpendicular to the LCMS, and $\delta$ the characteristic SOL width. Under the assumption that $\Gamma_{\perp}^{L C M S}$ and $\delta$ are independent of $l$, we get from Eq. (1) $\Gamma_{\|}=\Gamma_{\perp}^{L C M S} l / \delta$, with $l=0$ imposed at the SOL symmetry plane where $\Gamma_{\|}=0$.

In the heat transport equation 


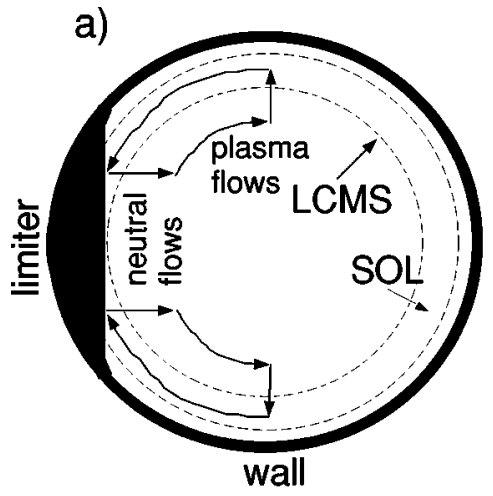

b)

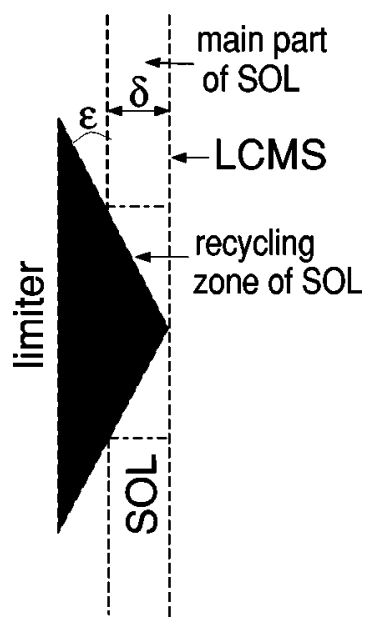

FIG. 1. The poloidal cross section and schematic view of particle flows at the plasma edge of a tokamak with a toroidal limiter (a) and characteristic zones of the limiter scrape-off layer (b). It is assumed that plasma particles flow into SOL homogeneously over the LCMS.

$$
\frac{d}{d l}\left(-\kappa_{\|} \frac{d T}{d l}+5 \Gamma_{\|} T\right)=\frac{q_{\perp}^{L C M S}}{\delta}
$$

the same temperature $T$ for electrons and ions is assumed and the Spitzer heat conductivity $\kappa_{\|}=A_{\kappa} T^{5 / 2}$ is dominated by the electron contribution. After one integration with the boundary condition $d T / d l=0$ at $l=0$ one gets

$$
-\frac{T^{5 / 2}}{T_{c}-T} d T=\frac{5 \Gamma_{\perp}^{L C M S}}{2 A_{\kappa} \delta} d l^{2} .
$$

Here $T_{c}=q_{\perp}^{L C M S} /\left(5 \Gamma_{\perp}^{L C M S}\right)$ is the maximum temperature in SOL which is determined by the condition that the heat flux is transported only by particle convection. Equation (3) can be integrated once more which gives an implicit relation between the temperatures at the symmetry plate, $T_{0}$, and at a certain $l, T_{l}$ :

$$
\begin{aligned}
& \frac{T_{c}^{5 / 2}}{2}\left(\ln \frac{1+\sqrt{T_{0} / T_{c}}}{1-\sqrt{T_{0} / T_{c}}}-\ln \frac{1+\sqrt{T_{l} / T_{c}}}{1-\sqrt{T_{l} / T_{c}}}\right) \\
& \quad=\frac{T_{0}^{5 / 2}-T_{l}^{5 / 2}}{5}+T_{c} \frac{T_{0}^{3 / 2}-T_{l}^{3 / 2}}{3}+T_{c}^{2}\left(\sqrt{T_{0}}-\sqrt{T_{l}}\right)+\frac{5 l^{2}}{4} \frac{\Gamma_{\perp}^{L C M S}}{A_{\kappa} \delta} .
\end{aligned}
$$

In real toroidal fusion devices the cross-field transport is localized on the low field side (LFS) and is not uniformly distributed poloidally. This is due to Shafranov shift of magnetic surfaces and the ballooning character of microinstabilities governing anomalous transport. In the limit case of a very strong localization the poloidal distribution of source terms on the right-hand sides (RHS) of Eqs. (1) and (2) can be approximated by $\delta$ function. The integration leads to the parallel fluxes of particles and energy independent of $l$ :

$$
\Gamma_{\|}=\Gamma_{\perp}^{L C M S} L / \delta, \quad-\kappa_{\|} d T / d l+5 \Gamma_{\|} T=q_{\perp}^{L C M S} L / \delta,
$$

where $L \equiv \pi q R$ is the distance from the symmetry plane to the limiter surface along magnetic field, $R$ the major radius of the LCMS, and $q$ the safety factor. Finally, one gets an equation similar to Eq. (4), but with the factor $5 \mathrm{Ll} / 2$ instead of $5 l^{2} / 4$ in the last term on the RHS.

\section{B. Recycling zone}

In this paper we do not consider states with a plasma detached from the limiter and, therefore, the loss of the parallel momentum due to charge exchange and elastic collisions can be neglected. In this case a well-known relation ${ }^{1}$ between the values of the plasma pressure at the symmetry plane and at the limiter surface can be applied:

$$
n_{0} T_{0}=2 n_{L} T_{L}
$$

where $T_{L}$ and $n_{L}$ are the plasma parameters near the limiter. Additionally, since the energy losses in the main part of the SOL are also small, the power coming into the SOL from the plasma confined volume is finally lost through two channels: (i) transferred to the limiter with the kinetic energy of charged particles and (ii) lost on the ionization of neutrals recycling from the limiter:

$$
4 \pi R \delta\left(\gamma T_{L}+E_{i}\right) n_{L} V_{s} \sin \psi=q_{\perp}^{L C M S} S_{L C M S} .
$$

Here $\gamma \approx 7.5$ is the heat transmission factor, $E_{i}$ the effective energy lost by the ionization of a recycling atom including its excitation, $V_{s}=\sqrt{2 T_{L} / m_{i}}$ the ion sound velocity acquired by the plasma ions near the limiter surface, $\psi \approx a /(q R)$ the pitch angle between magnetic field and toroidal direction with $a$ being the minor radius of the LCMS, and the LCMS area $S_{L C M S}=4 \pi^{2} a R$.

Equation (4) includes as a parameter the density of charged particle flux into the SOL from the confined volume, $\Gamma_{\perp}^{L C M S}$. In a stationary state, the total outflow, $\Gamma_{\perp}^{L C M S} S_{L C M S}$, is equal to the influx through the LCMS of neutrals produced at the limiter, $J_{n}^{L C M S}$. The neutral particles released from the limiter surface belong to two main fractions: (i) cold molecules desorbed with the surface temperature $T_{W} \ll T_{L}$ and velocity $V_{c} \approx 10^{3} \mathrm{~m} / \mathrm{s}$ and (ii) "hot atoms" with an energy of $T_{L}$, which are produced by reflection of ions. In addition, hot atoms are generated in the plasma volume by charge exchange of "cold atoms." In the present model we assume, however, that both components are atoms. The density $n_{c}$ of cold neutrals decays with the distance from the limiter, $x$, due to ionization by electrons and charge exchange with ions, with the rate coefficients $k_{i}$ and $k_{c x}$, respectively: 


$$
V_{c} \frac{d n_{c}}{d x}=-\left(k_{i}+k_{c x}\right) n n_{c} .
$$

The charge exchange of hot atoms leads to chaotic change of their velocity, and the variation of their density $n_{h}$ with $x$ can be modeled in a diffusion approximation: ${ }^{8,9}$

$$
\frac{d}{d x}\left(-D_{h} \frac{d n_{h}}{d x}\right)=-k_{i} n n_{h}+k_{c x} n n_{c}
$$

with the diffusivity $D_{h}=T / m_{i} /\left(k_{i}+k_{c x}\right) / n$; the last term in Eq. (8) gives the source density of hot atoms due to charge exchange of cold ones. The influxes of the cold and hot neutral components from the limiter surface are related to the plasma outflow density $j_{n}^{L}=n_{L} V_{s} \sin \psi \sin \varepsilon: \quad V_{c} n_{c}(0)=(1$ $\left.-R_{p}\right) j_{n}^{L}$ and $-D_{h} d n_{h} / d x(0)=R_{p} j_{n}^{L}$, where $R_{p}$ is the particle reflection coefficients. With these boundary conditions the equations above can be straightforwardly integrated (see, e.g., Refs. 2 and 9) providing $n_{c, h}$ as functions of the distance from the limiter surface, $x$ :

$$
n_{c}=\left(1-R_{p}\right) \frac{j_{n}^{L}}{V_{c}} \exp \left(-\sigma_{c} n_{L} x\right)
$$

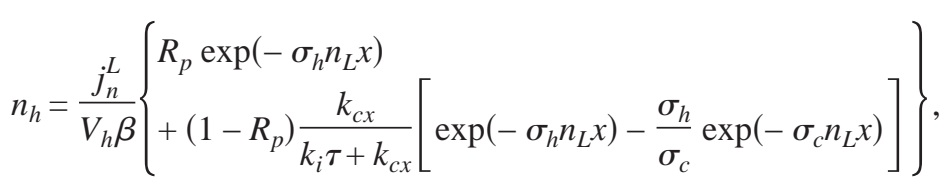

where $\sigma_{c}=\left(k_{i}+k_{c x}\right) / V_{c}$ and $\sigma_{h}=\sqrt{k_{i}\left(k_{i}+k_{c x}\right)} / V_{h}$ are the effective cross sections for the attenuation of cold and hot atoms, respectively, $\quad V_{h}=\sqrt{T_{L} / m_{i}}, \quad \beta=\sqrt{k_{i} /\left(k_{i}+k_{c x}\right)}, \quad$ and $\quad \tau=1$ $-T_{W} / T_{L}$. In $n_{h}$ the first term in brace gives the contribution from hot neutrals produced on the limiter surface through ion reflection and the second one, from those generated in the plasma by charge exchange of cold particles.

The total influx of neutrals into the confined volume is determined as follows:

$$
J_{n}^{L C M S}=4 \pi R \int_{0}^{\delta / \sin \varepsilon}\left[V_{c} n_{c}(s \sin \varepsilon)-D_{h} \frac{d n_{h}}{d x}(s \sin \varepsilon)\right] d s
$$

which gives

$$
\begin{aligned}
\Gamma_{\perp}^{L C M S} \equiv \frac{J_{n}^{L C M S}}{S_{L C M S}}= & \frac{4 \pi R \sin \psi}{S_{L C M S}} \frac{V_{s}}{\sigma_{h}} \\
& \times\left\{\begin{array}{l}
{\left[1-\exp \left(-\sigma_{c} n_{L} \delta\right)\right] \frac{\left(1-R_{p}\right) k_{i} \tau}{k_{i} \tau+k_{c x}} \frac{\sigma_{h}}{\sigma_{c}}} \\
+\left[1-\exp \left(-\sigma_{h} n_{L} \delta\right)\right] \frac{R_{p} k_{i} \tau+k_{c x}}{k_{i} \tau+k_{c x}}
\end{array}\right\} .
\end{aligned}
$$

Since $\sigma_{h} / \sigma_{c}=\beta V_{c} / V_{h}=\sqrt{k_{i} T_{W} /\left(k_{i}+k_{c x}\right) / T_{L}} \ll 1$, the first term in the brace, due to penetration of cold atoms, can be normally neglected. In particular, for the Tokamak Experiment for Technology Oriented Research (TEXTOR) parameters used below, this is less than $10 \%$ of the second one, due to hot atoms, for $n_{0} \geqslant 8 \times 10^{18} \mathrm{~m}^{-3}$, i.e., practically in the whole parameter range in question. In this case we obtain

$$
\Gamma_{\perp}^{L C M S} \approx \frac{4 \pi R \sin \psi}{S_{L C M S}} \frac{V_{s}}{\sigma_{n}}\left[1-\exp \left(-n_{L} \sigma_{n} \delta\right)\right] \frac{R_{p} k_{i}+k_{c x}}{k_{i}+k_{c x}},
$$

where $\tau \approx 1$ was used.

\section{SOL width}

An estimate for the SOL width $\delta$ follows by taking into account that the heat flux into SOL is transported through the LCMS by perpendicular plasma conduction and particle diffusion:

$$
q_{\perp}^{L C M S}=-\chi_{\perp} n_{0} \frac{d T}{d r}-3 D_{\perp} \frac{d n}{d r} T_{0},
$$

where $\chi_{\perp}$ and $D_{\perp}$ are the plasma heat and particle diffusivities. At the LCMS we assume $-d T / d r \approx T_{0} / \delta$ and $-d n / d r$ $\approx n_{0} / \delta$. By using Eqs. (6) and (11) one obtains

$$
\delta \approx \sqrt{\frac{2 L}{V_{s}} \frac{\chi_{\perp}+3 D_{\perp}}{\gamma+E_{i} / T_{L}}} .
$$

In the present model $\delta$ is actually the power flux width and we do not distinguish between $e$-folding lengths of density and temperature, which requires a further development. However, the simulations with the code EMC3-EIRENE, see the following section, show that under conditions in question these lengths do not differ very significantly and are in satisfactory agreement with the predictions from Eq. (12).

\section{RESULTS OF CALCULATION AND MODEL VALIDATION}

\section{A. Comparison with EMC3-EIRENE code simulations}

The set of equations (4)-(6), (10), and (12) allows us to determine the plasma characteristics in SOL. As control parameters we assume henceforth the heat influx into SOL, $q_{\perp}^{L C M S}$, and the plasma collisionality at the midplane, $\nu_{*}$ $=L / \lambda_{c}=\pi q R / \lambda_{c}$, where $\lambda_{c}$ is the electron mean free path length between Coulomb collisions. Computations have been performed for the conditions of additionally heated discharges in deuterium in the TEXTOR with $a=0.46 \mathrm{~m}, R$ $=1.75 \mathrm{~m}, S_{L C M S}=32 \mathrm{~m}^{2}, q=3.5, S_{L C M S} q_{\perp}^{L C M S} \approx 1 \mathrm{MW}, D_{\perp}$ $=\chi_{\perp} / 3=1 \mathrm{~m}^{2} / \mathrm{s}$. The ionization and charge-exchange rate coefficients, $k_{i}$ and $k_{c x}$, and the reflection coefficient $R_{p}$ are 


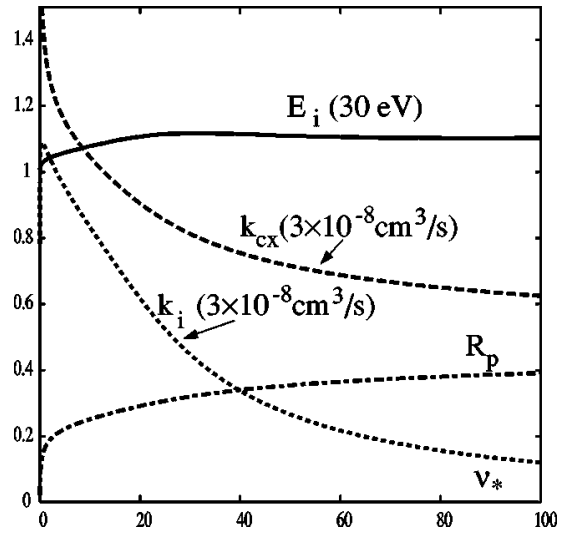

FIG. 2. The energy lost on ionization of a recycling atom, rate coefficients of atom ionization and charge exchange, and particle reflection coefficient from a carbon limiter surface vs the plasma collisionality.

computed according to the formulas in Ref. 2. Figure 2 shows the $\nu_{*}$ dependencies of the parameters $E_{i}, R_{p}, k_{i}$, and $k_{c x}$.

The comparison of plasma parameters computed by our analytical model and found in EMC3-EIRENE code simulations is done in Fig. 3. In Fig. 3(a) the code results are represented by the half of the sum of the electron and ion temperatures at the outer midplane, $T_{0}$, and near the limiter, $T_{L}$. Figure 3(b) represents the $\nu_{*}$ dependencies of the corresponding plasma densities, and Fig. 3(c) of the fraction of the neutral influx from the limiter, which is ionized in the SOL, $f_{S O L}$, and of the fraction of the total power lost on ionization, $f_{\text {power }}=E_{i} /\left(\gamma T_{L}+E_{i}\right)$. One can see that for all presented characteristics the analytical results reproduce well qualitative features of the code simulations. The quatitative difference does not exceed $30 \%$.

A very striking feature observed both in analytical and code calculations is the weak variation of $f_{S O L}$ with $\nu_{*}$. According to the present model

$$
f_{S O L}=1-\frac{1}{u}\left\{\begin{array}{c}
{\left[\left(1-e^{-\sigma_{c} / \sigma_{h} u}\right)\right] \frac{\left(1-R_{p}\right) k_{i} \tau}{k_{i} \tau+k_{c x}} \frac{\sigma_{h}}{\sigma_{c}}} \\
+\left(1-e^{-u}\right) \frac{R_{p} k_{i} \tau+k_{c x}}{k_{i} \tau+k_{c x}}
\end{array}\right\},
$$

where $u=n_{L} \sigma_{h} \delta$; for $\nu_{*} \gtrsim 10$ practically all cold neutrals are ionized in SOL and $f_{S O L}$ is well described by the reduced expression

$$
f_{S O L}=1-\frac{1-e^{-u}}{u} \frac{R_{p} k_{i}+k_{c x}}{k_{i}+k_{c x}} .
$$

In this case, $f_{S O L}$ always exceeds $\left(1-R_{p}\right) k_{i} /\left(k_{i}+k_{c x}\right)$ and should approach 1 with $u \rightarrow \infty$. However, very large $u$ cannot be reached under conditions in question because $\sigma_{h}$ reduces fast when $T_{L}$ is diminished to a level comparable with the hydrogen ionization energy. For the particular conditions $u$ $\lesssim 0.9, \quad\left(1-e^{-u}\right) / u \gtrsim 0.66 \quad$ and $\quad f_{S O L} \lesssim\left[\left(1-0.66 R_{p}\right) k_{i}\right.$ $\left.+0.34 k_{c x}\right] /\left(k_{i}+k_{c x}\right)$. Additionally, the reflection coeffcient $R_{p}$ increases with decreasing temperature and relatively more hot atoms are produced on the limiter. This further sup-

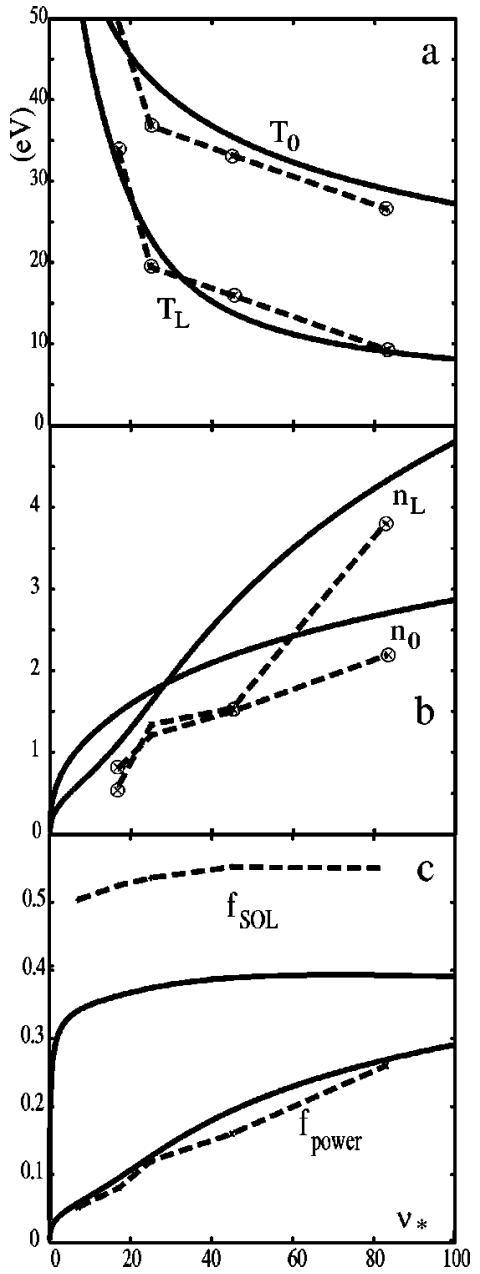

FIG. 3. Comparison of the results of the present improved 2-point model (solid curves) and the code EMC3-EIRENE simulations (broken curves): plasma density (a) and temperature (b) at the SOL midplane and at the limiter; the fractions of the neutral flux from the limiter ionized in the SOL, $f_{S O L}$, and of the power lost on ionization, $f_{\text {power }}$, (c), vs the plasma collisionality.

presses the tendency of $f_{S O L}$ to increase with rising collisionality.

With increasing plasma density and collisionality other important effects neglected in the present model are involved, e.g., charge-exchange power loss and friction between ions and neutrals. However, under the conditions in question, with $T_{L} \gtrsim 7-8 \mathrm{eV}$, the results of EMC3-EIRENE simulations justify this neglect and predict that the losses from the ion component with the charge-exchange do not exceed several percent of the total power transferred into the SOL. The conservation of the total momentum given by Eq. (5) is also satisfied with the same accuracy. Finally, EMC3EIRENE simulations did not include impurity radiation and it is difficult to judge what is the consequence of its absence in the present analytical model.

Concerning the SOL width $\delta$, the code simulations predict that at low collisionality the $e$-folding lengths of the plasma density and temperature, $\delta_{n}$ and $\delta_{T}$, respectively, are practically the same and of $1.6 \mathrm{~cm}$. For these conditions Eq. (12) gives $1.9 \mathrm{~cm}$. The code results show that with increasing collisionality $\delta_{n}$ changes weakly but $\delta_{T}$ grows up to $3.8 \mathrm{~cm}$ 
at $\nu_{*}=45$. The latter tendency is reproduced by our model; however, it predicts $\delta=2.7 \mathrm{~cm}$. Remarkably, this value coincides with the code prediction for the value averaged between $\delta_{n}$ and $\delta_{T}$.

\section{B. Comparison with corrected standard 2-point model}

In Ref. 1 the standard 2-point model, which neglects convective energy losses, has been corrected by taking those into account implicitly, through the reduction of the conductive parallel heat flux in the SOL main part by a factor $f_{\text {cond }}<1$. For comparison of predictions from our model and the corrected standard 2-point model we choose as a figure of merit the ratio of plasma temperatures at the SOL symmetry plane and near the limiter, $\theta=T_{0} / T_{L}$.

In order to come to a formula for $\theta$ prescribed by the corrected standard 2-point model we omit the term $5 \Gamma_{\|} T$ on the left-hand side of Eq. (2) but reduce the source term on the RHS by the factor $f_{\text {cond }}$. Under the assumption of $f_{\text {cond }}$ independent of $l$, the integration leads to

$$
T_{L}=\left(T_{0}^{7 / 2}-\frac{7 q_{\perp}^{L C M S} f_{\text {cond }}}{4 A_{\kappa} \delta} L^{2}\right)^{2 / 7} .
$$

If $T_{0}$ is at least slightly greater than $T_{L}$ then

$$
\frac{q_{\perp}^{L C M S} L}{\delta} \approx \frac{4 A_{\kappa} T_{0}^{7 / 2}}{7 L f_{\text {cond }}} .
$$

From Eqs. (5) and (6) we obtain

$$
\frac{q_{\perp}^{L C M S} L}{\delta}=\frac{\gamma n_{0} T_{0} V_{s}}{2\left(1-f_{\text {power }}\right)} .
$$

By combining these relations and taking into account ${ }^{10} \kappa_{\|}^{e}$ $\equiv A_{k} T_{0}^{5 / 2}=3.16 n_{0} T_{0} \tau_{e} / m_{e}$ and $\lambda_{c}=\tau_{e}\left(2 T_{0} / m_{e}\right)^{1 / 2}$ one gets

$$
\sqrt{\frac{T_{0}}{T_{L}}}=\frac{7 \gamma}{4 \times 3.16} \frac{L}{\lambda_{c}} \sqrt{\frac{m_{e}}{m_{i}}} \frac{f_{\text {cond }}}{1-f_{\text {power }}}
$$

or

$$
\theta \simeq \frac{\nu_{*}^{2}}{100 A_{i}}\left(\frac{f_{\text {cond }}}{1-f_{\text {power }}}\right)^{2},
$$

where $A_{i}$ is the ion atomic weight.

Thus $\theta$ essentially depends on $f_{\text {cond }}$ and one needs an approach to estimate this. In particular, one can use its value at the outboard midplane, which can be found from the following relation:

$$
5 T_{0} n_{L} V_{s}\left(1-f_{\text {SOL }}\right)=\gamma T_{L} n_{L} V_{s} \frac{1-f_{\text {cond }}}{1-f_{\text {power }}} .
$$

This gives

$$
f_{\text {cond }}=1-\frac{5}{\gamma}\left(1-f_{\text {power }}\right)\left(1-f_{\text {SOL }}\right) \theta
$$

and, in combination with Eq. (14), a quadratic equation for $\sqrt{\theta}$. Finally

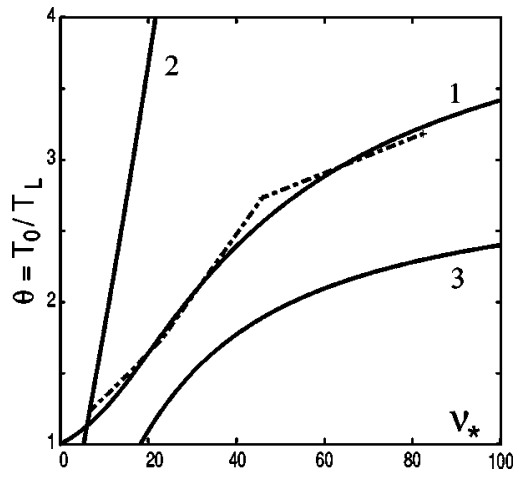

FIG. 4. The ratio of temperatures at the SOL symmetry plane and near the limiter vs the plasma collisionality in SOL computed in the EMC3-EIRENE code simulations (broken curve), by the present improved 2-point model (solid curve 1), standard 2-point model, and corrected 2-point model (curve 3) for deuterium plasmas in TEXTOR.

$$
\theta=\left[\frac{\sqrt{\frac{20}{\gamma} \frac{1-f_{\text {SOL }}}{1-f_{\text {power }}}+\frac{100 A_{i}}{\nu_{*}^{2}}}-\frac{\sqrt{100 A_{i}}}{\nu_{*}}}{\frac{10}{\gamma}\left(1-f_{\text {SOL }}\right)}\right]^{2} .
$$

This relation includes $f_{S O L}$ and $f_{\text {power }}$. According to Fig. 3, $f_{S O L}$ does not significantly depend on the collisionality and $f_{\text {power }} \ll 1$ for conditions in question. Henceforth we use $f_{\text {SOL }}=0.4$ and $f_{\text {power }}=0.15$.

Figure 4 shows the $\nu_{*}$ dependence of $\theta$ found in the EMC3-EIRENE code simulations (broken curve), by applying the present improved 2-point model (solid curve 1), standard 2-point model, i.e., Eq. (14) with $f_{\text {cond }}=1$ (curve 2), and corrected standard 2-point model given by the Eq. (15) (curve 3) for deuterium plasmas. One can see that our model perfectly reproduces the code results, while the standard 2 -point model gives strongly overestimated values of $\theta$. Although the corrected standard 2-point model gives somewhat low values, it qualitatively reproduces the saturation of $\theta$ magnitude at high collisionality. By applying Eq. (15) one should, however, keep in mind that $\theta$ predicted by this depends essentially on $f_{S O L}$. In particular, our computations show that $f_{S O L}$ varies essentially with the heat flux into the SOL.

\section{Interpretation of results}

In order to understand the cause for the saturation of $\theta$ at large collisionality we have to take into account the variation of the plasma parameters far and near the limiter shown in Figs. 3(a) and 3(b). At a small density and low collisionality, SOL recycling zone is transparent for neutrals and particle convection dominates in the heat transport. Additionally, the plasma temperature in SOL is high enough and the parallel heat conduction, $\sim T^{5 / 2}$, is large. As a result the temperature variation along SOL is small and $\theta \approx 1$. With increasing collisionality, the probability of neutral ionization in SOL also increases and the contribution of convection to the heat flux in the SOL main part drops. Therefore with decreasing plasma temperature the heat conduction should transport more power, which requires larger parallel temperature gra- 
dient, i.e., $\theta$ grows. When $n_{0}$ reaches a level of 1.5 $\times 10^{19} \mathrm{~m}^{-3}$, the temperature near the limiter drops below the ionization energy of hydrogen neutrals, $I_{H}=13.6 \mathrm{eV}$, and the attenuation cross section $\sigma_{n} \sim \sqrt{k_{i}} \sim \exp \left[-I_{H} /(2 T)\right]$ reduces promptly with the temperature. Therefore neutrals can penetrate into the confined plasma volume, the level of convective energy in SOL main part stabilizes, and the $\theta$ value saturates.

By concluding this section we note that the computations with the particle and heat sources strongly localized on LFS (see the last paragraph of Sec. II) results in a $\nu_{*}$ dependence of $\theta$, which saturates at the same level as for homogeneously distributed source. This saturation occurs, however, already at smaller values of $\nu_{*}$.

\section{CONCLUSION}

An improved 2-point model for a limiter SOL is proposed, which self-consistently takes into account the convective energy losses along field lines in the main SOL part. The model predicts in quantitative agreement with the code simulations the characteristic plasma parameters in the SOL of TEXTOR and the saturation of the ratio between electron temperatures far and near the limiter surface with increasing plasma collisionality. An interpretation of this phenomenon, being in striking contradiction with the standard 2-point model, but in qualitative agreement with corrected 2-point model, is proposed: when the temperature near the limiter drops below the hydrogen ionization energy, neutrals can penetrate into the confined plasma volume and convective energy transport remains significant even at the highest plasma density.

${ }^{1}$ P. C. Stangeby, The Plasma Boundary of Magnetic Fusion Devices (Institute of Physics, Bristol, 2000), p. 221.

${ }^{2}$ A. V. Nedospasov and M. Z. Tokar, in Review of Plasma Physics, edited by B. B. Kadomtsev (Consultants Bureau, New York, 1990), Vol. 18, p. 68.

${ }^{3}$ B. J. Braams, Contrib. Plasma Phys. 36, 276 (1996).

${ }^{4}$ T. D. Rognlien, B. J. Braams, and D. A. Knoll, Contrib. Plasma Phys. 36, 105 (1996).

${ }^{5}$ M. Baelmans, D. Reiter, and R. R. Weynants, Contrib. Plasma Phys. 36, 117 (1996).

${ }^{6}$ Y. Feng, F. Sardei, P. Grigull, K. McCormick, J. Kisslinger, D. Reiter, and Y. Igitkhanov, Plasma Phys. Controlled Fusion 44, 611 (2002).

${ }^{7}$ M. Kobayashi, Y. Feng, F. Sardei, K. H. Finken, D. Reiser, and D. Reiter, Nucl. Fusion 44, 64 (2004).

${ }^{8}$ B. Lehnert, Nucl. Fusion 8, 173 (1968).

${ }^{9}$ M. Z. Tokar, Plasma Phys. Controlled Fusion 35, 1119 (1993).

${ }^{10}$ S. I. Braginski, in Review of Plasma Physics, edited by M. Leontovich (Consultants Bureau, New York, 1963), Vol. 1, p. 193. 